\title{
The Safety and Efficacy of the Interleukin-1 Antagonist Anakinra in Severe Cases of COVID-19- A Systematic Review and Meta-analysis.
}

Manoj Kumar Reddy Somagutta ( $\square$ manojleo.go@gmail.com )

Larkin Community Hospital Inc.

Maria Kezia Lourdes Pormento

Larkin Community Hospital Inc.

Pousette Hamid

California Institute of Behavioral Neurosciences and Psychology

Alaa Hamdan

Larkin Community Hospital Inc.

Muhammad Adnan Khan

Larkin Community Hospital Inc.

Rockeven Desir

Larkin Community Hospital Inc.

\section{Rupalakshmi Vijayan}

Larkin Community Hospital Inc.

\section{Saloni Shirke}

Larkin Community Hospital Inc.

Rishan Jeyakumar

Larkin Community Hospital Inc.

\section{Zeryab Dogar}

Larkin Community Hospital Inc.

\section{Sarabjot Singh Makkar}

Larkin Community Hospital Inc.

\section{Prathima Guntipalli}

Larkin Community Hospital Inc.

\section{Neguemadji Ngardig Ngaba}

Larkin Community Hospital Inc.

\section{Manasa Sindhura Nagineni}

Larkin Community Hospital Inc.

\section{Trissa Paul}

Larkin Community Hospital Inc.

\section{Enkhmaa Luvsannyam}


Larkin Community Hospital Inc.

\section{Chala Riddick}

Larkin Community Hospital Inc.

\section{Sukrut Pagad}

Larkin Community Hospital Inc.

\section{Marcos Sanchez-Gonzalez}

Larkin Community Hospital Inc.

\section{Systematic Review}

Keywords: COVID-19, Anakinra, Interleukin, IL-1 Febrile Inhibitor, SARS CoV 2, Kineret

Posted Date: November 19th, 2020

DOI: https://doi.org/10.21203/rs.3.rs-111495/v2

License: (c) (i) This work is licensed under a Creative Commons Attribution 4.0 International License. Read Full License 


\section{Abstract}

This study aims to assess anakinra's safety and efficacy for treating severe coronavirus disease in 2019 (COVID-19). PubMed, Google Scholar, Cochrane Library, Embase, Scopus, medRxiv, and bioRxiv were searched. Three retrospective studies and five case series involving 3,274 adult patients with severe COVID-19 were included, 621 treated with anakinra (whether administered alone or in combination with other drugs) and 1,565 in the control group arm. All-cause mortality of severe COVID-19 patients among the anakinra group was $20 \%(16 / 81)$, which was lower than that in the control group $(65 \% ; 39 / 60)$. The difference was statistically significant [hazard ratio $(H R)=0.13,95 \%$ confidence interval $(\mathrm{Cl}) 0.06-0.29$, $12=0 \%]$. The mechanical ventilation requirement with OR $0.57(0.11-2.84,12=87 \%)$ was not significantly better compared to the control group. For the safety of anakinra, we evaluated thromboembolism risk and liver enzyme elevation. Thromboembolism risk with OR: 1.48 (0.55- 3.99, I2=0\%) and elevation in liver transaminases with OR $0.67(0.11-3.93,12=66 \%)$ were not statistically significant over the control group. However, these non-significant differences between the anakinra and control groups may have been the result of baseline characteristics of the intervention group, and further studies are essential in evaluating anakinra's safety profile.

\section{Introduction}

In December 2019, an outbreak of emerging severe acute respiratory syndrome coronavirus 2 (SARS-CoV2) disease (COVID-19) in China was brought to international notice and declared a pandemic by the World Health Organization (WHO) on March 11, 2020 [1]. As of October 2020, there are a total of 8,128,524 cases and 218,986 deaths attributed to COVID-19 in the United States of America [2]. The ongoing pandemic has presented a devastating effect on people's social, and economic, well-being by infecting over four million people worldwide. The alarmingly rising fatality rates demand an early intervention to guard and safeguard the vulnerable population. The need for innovative and effective treatment modalities is crucial as more people succumb to the virus [3]. Presently, there are no specific treatments or measures recommended to prevent or treat COVID-19. Clinical management is currently limited to supportive care [3]. Understanding the disease pathophysiology and targeting therapy towards the molecular processes is key in identifying effective therapeutic interventions. Coronaviruses are a group of RNA viruses with envelopes commonly present in mammals and birds, causing respiratory or gastrointestinal diseases with a few neurological debilities or hepatitis [4]. Infections are transmitted mainly via respiratory and fecal-oral routes and manifest [5].

The inflammatory response in COVID-19 plays a fundamental role in the high mortality seen in patients. The virus is recognized by the innate immune system leading to the recruitment of immune cells and the production of various cytokines. Excessive production of these inflammatory cytokines causes a phenomenon termed the cytokine storm, which leads to the development and exacerbation of ARDS or extrapulmonary multiple organ failure. Many cytokines and chemokines have been implicated in the 
pathogenesis of this hyperinflammatory response [6]. This cytokine profile resembles secondary hemophagocytic lymphohistiocytosis (sHLH), a hyperinflammatory syndrome resulting in fulminant multiorgan failure. The underlying etiologies of sHLH include viral infections and sepsis [7]. The H-score is a good valid indicator of hemophagocytic syndromes including sHLH. Measurements include immunosuppression status, temperature, organomegaly, number of cytopenias, ferritin levels, triglycerides, fibrinogen, serum aspartate aminotransaminase, and hemophagocytosis features on bone marrow aspirate [8]. It has been suggested that all severe COVID-19 patients should have their $\mathrm{H}$-score evaluated and used as a tool to guide therapeutics. When elevated and indicative of reactive HLH, immunosuppressives may be an option including steroids, intravenous immunoglobulin, selective cytokine inhibitors (i.e anakinra, tocilizumab) and medications inhibiting JAK pathways [7].

This systematic review and meta-analysis aims to identify the role of anakinra, an interleukin-1 receptor antagonist (IL-1Ra) in the treatment of COVID-19. Multiple studies have been performed on this drug, but the direct effects have not been reviewed in a large-scale study. We will be reviewing currently available evidence and methodology to determine the safety and efficacy of anakinra therapy in COVID-19 patients. We will analyze how anakinra modifies the disease duration and determine if it prolongs, reduces, or has no effect on viremia. Since anakinra is suspected of inhibiting a key cytokine in the pathogenesis of the hyper inflammation, the study will evaluate its therapeutic role in disease severity and secondary outcomes (i.e., the need and duration of mechanical ventilation, respiratory failure, death, etc.). Furthermore, this review will determine what dosage of the drug has shown optimal efficacy and safety in the treatment of COVID-19.

\section{Methods}

\section{Data Sources and Search Selection}

This systematic review and meta-analysis was conducted based on pre-registered protocol in 'Prospero' (CRD42020203708). The research was done through database searches looking for different studies to answer the clinical question to merit a systematic review and meta-analysis. A systematic review was performed according to the preferred reporting items for systematic reviews and metaanalysis guidelines (PRISMA). Institutional Review Board (IRB) approval was waived for this study because it reviews the current literature. The literature research was started on the 20th of July 2020 and continued for five consecutive days till 25th July 2020. The research was conducted on PubMed, Medline, Cochrane Library, Google scholar, Cinahl, Clinicaltrials.gov, Embase, Scopus, medRxiv, and bioRxiv. A total of 269 articles with the potential to be included in the study were identified.

\section{Study strategy}

The search for articles was done using the keywords, MeSH terms, or synonyms to collect many studies. Different keywords, MeSH terms, and synonyms including 'COVID-19', 'Anakinra', 'IL-1 Febrile Inhibitor', 'Anakinra in SARS CoV 2', 'IL-1Ra', 'Urine-Derived IL1 Inhibitor', 'Urine Derived IL1 Inhibitor, 'IL 1 Inhibitor', 'Urine', 'Urine IL-1 Inhibitor', 'Interleukin 1 Inhibitor', 'Urine', 'Antril', 'Kineret', combined with Boolean 
operators OR/AND were used. Studies were chosen by said keywords and tabulated. After screening, the duplicate articles were removed and second-time studies that did not fulfill the inclusion criteria. At last, examinations that satisfied the qualification rules were included and affirmed through accord.

\section{Inclusion/exclusion criteria}

Primary literature was searched using the PICO design and strategy. The study incorporated all patients tested positive for COVID-19 (age > 18, all sexes, all nationalities, all geographical areas, multicultural background and ethnicity), various control groups (no treatment, treatment according to the hospital guidelines, $\mathrm{NIH}$ guidelines), as well as the impact of using anakinra in the clinical outcome of patients (improvement, deterioration and progression to the complications) and drug efficacy. Although the research was conducted, searching for different study designs, case series, observational studies, controlled randomized and non-randomized clinical trials, published and pre-prints were included to address a significant part of gray literature and reduce the risk of selection bias.

\section{Data extraction and the Risk of bias assessment}

All data from studies were extracted by 2 independent reviewers onto prespecified forms. Pooled mortality was the main outcome measured. Hazard Ratio (HR) and the $95 \% \mathrm{Cl}$ were extracted from each study. We selected HR as an effect estimate. We calculated the odds ratio from secondary outcomes, including the requirement for mechanical ventilation, thromboembolic events, and elevation of liver transaminases. We also extracted baseline information of the participants, sample size, and follow-up duration.

Three authors independently assessed the risk of bias qualitatively for selecting the study groups, the comparability of the groups, and the ascertainment of the outcome of cohort studies using the Newcastle-Ottawa Scale (NOS) Checklist. We used the CARE Checklist (2013) for accuracy, transparency, and usefulness of case series studies. Disagreements were resolved by discussion or with a fourth author. We evaluated the studies for the possibility of publication bias and selective reporting bias by assessing the symmetry of funnel plots visually and more objectively with the Egger's test. Due to the number of studies included, publication bias and selective outcome reporting are difficult to exclude or verify. The funnel plot's asymmetry may also indicate a difference in the methodological quality and intervention effects estimated in the studies with smaller samples.

\section{Statistical Analysis}

The analysis was done using Review Manager (Version 5.3; The Cochrane Collaboration; London, United Kingdom). All the outcomes reviewed were dichotomous; point estimates are expressed as odds ratio (OR) with the $95 \%$ confidence interval (Cl). A random-effects model was applied when data were considered as heterogeneous, respectively. Results were also displayed graphically by the creation of forest plots. The heterogeneity of outcomes was calculated using the $I^{2}$ statistic. The $I^{2}$ value of $0 \%-40 \%$ was considered to be not important; $30 \%$ to $60 \%$ as moderate heterogeneity; $50 \%-90$ as substantial 
heterogeneity, and $75 \%$ to $100 \%$ as considerable heterogeneity. The meta-analysis was performed using the Mantel Hazel method. A p-value $<0.05$ was considered to be statistically significant.

\section{Results}

\section{Literature Search Results}

This literature search lasted for five consecutive days, utilizing various research search engines -a total of 269 articles which have been identified. The independent researchers found various studies from randomized controlled control studies, case series, case reports, and cohort studies. The PRISMA flowchart (Figure 1) summarizes the literature search.

\section{Study Characteristics}

The search strategy yielded 269 references after excluding 96 duplicate articles, 104 articles were subsequently screened, and eight articles were identified for full-text review for eligibility. Among them, three were observational studies, and five were case series. The three retrospective cohort studies were designed to compare anakinra's clinical efficacy, thus were included in the meta-analysis. This metaanalysis revealed 12 articles ( 3 cohort studies- 5 case series- 4 case reports) deemed relevant for full-text review. A total of 3,274 adult patients with severe COVID-19, including 621 in the anakinra (whether administered alone or in combination with other drugs) and 1,565 in the control group arm, were included. The mean age was 55.25 years old; 9 studies had a mean age of more than 50 years old, and three studies had a mean age less than 50 years old.

The characteristics of the included cohort studies and case-series are available in supplementary table S1. We encountered no multicenter studies, and three observational studies were single-center studies. Three studies were conducted in different geographical locations such as France [9], Italy [10] and the U.S [11]. Only two studies had matched the study and control group in terms of age, sex and disease severity. The frequency of administration ranged from once a day to four times a day with a dosage of $100 \mathrm{mg}$ to $300 \mathrm{mg}$ among the cohorts and the case series

\section{Outcomes}

Pooled analysis of the three included studies $[9,10,12]$ revealed that the all-cause mortality rate of patients with COVID-19 in the anakinra treatment group was 20\% (16/81), which was lower compared with the control group $(65 \% ; 39 / 60)$. The difference was statistically significant [HR $=0.13,95 \%$ confidence interval $(\mathrm{Cl}) 0.06-0.29, \mathrm{I}^{2}=0 \%$ ]. The mechanical ventilation requirement was similar between anakinra and control groups [OR $0.57\left(0.11-2.84, \mathrm{I}^{2}=87 \%\right)$ ]. For the safety of anakinra administration, we evaluated the odds ratios of thromboembolic events and liver enzyme elevation, finding non-significance: thromboembolism risk [OR $1.48\left(0.55-3.99, I^{2}=0 \%\right)$ ] and elevation in liver transaminases [OR $0.67(0.11$ $\left.\left.3.93, \mathrm{I}^{2}=66 \%\right)\right]$. 
Mortality as a clinical outcome was also addressed in two case-series studies involving anakinra administration to COVID-19 patients. In case series studies by Dimopoulos et al, and Navarro-Millán et al, mortality on day 28 was estimated at $37.5 \%$ (3/8) and 9\% (1/11), respectively. No mortality was noted in the studies of Day et al, Aouba et al, and Pontali et al Navarro-Millan et al noted that early initiation of the drug before requiring supplemental 02 therapy prevented worsening in clinical status (i.e., need for mechanical ventilation). Out of four mechanically ventilated patients receiving anakinra, two patients were discharged, and one patient died [11]. In the studies of Aouba et al and Navarro-Millan et al an increase in liver transaminases in $6(75 \%)$ and $3(27.3 \%)$ patients were noted. Dimopoulos reported none of the patients had an increase in liver transaminases.

\section{Study or Subgroup analysis}

Subgroups were created based on patient demographic data (age, gender, survival outcome and comorbidities). A total of 621 patients are in the anakinra group, while 1,565 are in the control group. Three studies were included in the subgroup analysis $[9,10,12]$. Male patients were 396 vs 975 in the anakinra and control group respectively [OR $1.17(0.96-1.43, p=0.13)$ ]. While female patients were $197 \mathrm{vs}$ 590 [OR $0.85(0.70-1.05, p=0.13)$ ]. Survival outcome (No ICU, Alive, No IMV) was 545 vs 1456 patients; [OR $193(1.27-2.93, p=0.002)]$, in the anakinra group and control group, respectively.

Clinical comorbidities such as hypertension were found in 327 vs 734 patients in the anakinra arm and control group with [OR $1.36(1.12-1.65, p=0.002)$ ]. Diabetes is reported in 198 vs 506 patients [OR 1.09 $(0.89-1.33, p=0.430)$ ]. Cardiovascular comorbidities are found in 70 vs 197 patients; [OR 0.88 (0.66-1.19, $\mathrm{p}=0.41)]$, in the anakinra group and control group, respectively.

\section{Risk of Bias, Publication Bias and Overall Certainty of Evidence}

The studies overall had moderate to good methodological quality. Having a limited number of studies included in the meta-analysis $(n=3)$, approaches to evaluate the publication bias might have limited efficacy. However, the attempt to report the publication bias showed the funnel plot (Figure 2.0), suggesting minimal publication bias among the included studies. The risk of bias assessment was done using the Cochrane Risk Bias Assessment (Table 1) and Joanna Briggs Institute Appraisal Too for the cohort studies and case series, respectively.

Table 1. Risk of bias assessment for included cohort studies (Cochrane Risk Bias Assessment) 


\begin{tabular}{|c|c|c|}
\hline & $\begin{array}{l}\text { Cavalli } \\
\text { et al. }\end{array}$ & $\begin{array}{l}\text { Huet } \\
\text { et } \\
\text { al. }\end{array}$ \\
\hline $\begin{array}{l}\text { Was selection of exposed and non-exposed cohorts drawn from the same } \\
\text { population? }\end{array}$ & Low & Low \\
\hline Can we be confident in the assessment of exposure? & Low & Low \\
\hline $\begin{array}{l}\text { Can we be confident that the outcome of interest was not present at the start } \\
\text { of study? }\end{array}$ & Low & Low \\
\hline $\begin{array}{l}\text { Did the study match exposed and unexposed for all variables that are } \\
\text { associated with the outcome of interest or did the statistical analysis adjust } \\
\text { for these prognostic variables? }\end{array}$ & $\begin{array}{l}\text { High } \\
\text { risk }\end{array}$ & Low \\
\hline $\begin{array}{l}\text { Can we be confident in the assessment of the presence or absence of } \\
\text { prognostic factors? }\end{array}$ & Low & Low \\
\hline Can we be confident in the assessment of outcome? & $\begin{array}{l}\text { High } \\
\text { risk }\end{array}$ & $\begin{array}{l}\text { High } \\
\text { risk }\end{array}$ \\
\hline Was the follow up of cohorts adequate? & Low & Low \\
\hline Were co-interventions similar between groups & Low & Low \\
\hline
\end{tabular}

\section{Discussion}

Respiratory failure and ARDS have been reported to be the main cause of death in COVID-19 patients followed by sepsis and multiorgan failure $[13,14]$. Prior to the development of ARDS, COVID-19 patients without clinical manifestations become hypoxic through preliminary endothelial damage through inflammatory cytokine release and unusually large von Willebrand factor multimers (ULVWF) coexisting with platelet over-activation. This asymptomatic hypoxemia can be a determining feature of prognosis. Furthermore, endotheliitis through venous thromboembolism and microthrombi leads to the characteristic coagulation overactivation, coagulopathy and elevated permeability of the blood vessels due to endothelial damage in Covid-19, as seen in parallel with the H1N1 molecular pathogenesis and is also characteristic of sHLH, ARDS and multiple organ dysfunction syndrome (MODS) [14]. Delving into the molecular biology of this pathway, ARDS is primarily caused by a cytokine storm, an unregulated systemic release of inflammatory cytokines [15]. Cytokine Release Syndrome (CRS), also ubiquitously referred to as cytokine storm syndrome (CSS), is the process by which an elevation of inflammation and damage to the endothelium occurs and precipitates the excessive activation of the coagulation pathways as well as disrupts the integrity of the vascular permeability. A characteristic set of markers determine cytokine storm intensity, a mortality marker composed of elevated GCSF, PAF, thrombin-induced MIF, TNF, cystatin C, IL-1, IL-6, ferritin leading to immune dysregulation injury that causes endothelial damage $[14,16]$. CRS induced dysregulation of immune responses cause epithelial and endothelial cell apoptosis 
and vascular leakage, suboptimal T cell responses, dysfunctional and overactive macrophages infected with SARS-CoV-2 and dysfunctional tissue homeostasis, all of which contribute to the pathogenesis and severity of macrophage activation storm (MAS) leading to ARDS and MODS [14-18]

MAS is indirectly induced by IL-1 [18]. This may be the target point to prevent ARDS development by inhibition of IL-1 and resultant MAS and ARDS. Additionally, increased IL-1 release in viral infections results in lung and tissue inflammation, fever and fibrosis. An over-expression of IL-1 is pathognomonic of SARS-CoV-2 by activating transcription factor, nuclear factor, activator protein 1 and activating factor 2. This virus binds to Toll-like receptors (TLRs) to stimulate pro-IL-1 production and inflammasome activation regulating cells of both the innate and adaptive immune system and produces specific immune responses. IL1-b, thus produced, results in the inflammation of the lungs, fever and fibrosis to lead to respiratory complications in the infected host [19].

Anakinra is a recombinant, non-glycosylated form of the human interleukin-1 receptor antagonist (Recombinant human interleukin-1 receptor antagonist (IL-1Ra) [20]. It is produced by recombinant DNA technology by using an $E$. Coli bacterial expression system. It is different from native human IL-1Ra, as it has an additional single methionine residue at its amino terminus [20]. It acts by blocking the biological activity of IL-1 $a$ and IL-1 $\beta$ (pro-inflammatory cytokines) by competitive inhibition of IL-1 binding to the interleukin-1 type I receptor (IL-1R1). Currently, intravenous and subcutaneous routes are available with a 95\% bioavailability and a half-life of 3-7 hours [21]. It has been used successfully in treating macrophage activation syndrome caused by various inflammatory conditions, including Still's disease, HLH, and active rheumatoid arthritis [22]. IL-1 is produced as a result of physiological response to inflammatory stimulus and immunological conditions [23]. IL-1 $\mathrm{a}$ and IL-1 $\beta$ are activated through inflammasome and proinflammatory cytokines that mediate many cellular responses. They also increase nitric oxide production, prostaglandin, adhesion molecules, thromboxane, and histamine [23]. These factors also contribute to the "cytokine storm" implicated in the pathogenesis of COVID-19. This mechanism of action may be helpful in COVID-19, according to previous studies. Various clinical trials are being conducted that support this rationale for targeting hyper inflammation in COVID-19 with anakinra. Outcomes are promising, but varied use and dosage and outcomes have been noticed in the various trials [20].

To the best of our knowledge, this is the first meta-analysis investigating the effect of anakinra on clinical outcomes of patients with severe COVID-19. Based on the analysis of three retrospective studies $[9,10,11]$, it was found that anakinra could provide additional benefit for improving the mortality outcome of severe COVID-19. However, it was found that anakinra could not provide any additional benefit for other clinical outcomes of severe COVID-19. In the study by Huet et al, of 96 patients, the mortality was $25 \%(13 / 52)$ compared to $73 \%(32 / 44)$ in control, demonstrating that overall mortality favors anakinra administration. This was further demonstrated in the study by Cavalli et al, where mortality was $10 \%(3 / 29)$ in the anakinra group compared to $44 \%(7 / 16)$ in the control group. In a smaller size study of Dimopoulos, Navarro-Millan, the mortality was reported to be $37.5 \%$ (3/8) and $9.09 \%(1 / 11)$, respectively. In contrast, the mortality rate favored the control group in Narain et al with an $\mathrm{HR}$ at $95 \% \mathrm{Cl} 2.12$ (1.68-2.66). In the study by Huet et al, of the 96 patients, the need for mechanical ventilation was $25 \%(13 / 52)$ than $73 \%$ 
(32/44) in control, demonstrating that the overall need for mechanical ventilation favors anakinra administration. Cavalli et al noted similar findings with $17 \%(5 / 29)$ in the anakinra group and $6 \%(1 / 16)$ in the control group. In a smaller size study of Dimopoulos et al and Navarro-Millan et al, the need for mechanical ventilation was reported to be $87.5 \%(7 / 8)$ and $36.4 \%(4 / 11)$, respectively. Several studies reported an overall decrease in the inflammatory marker levels, such as C- reactive protein (CRP) levels, ferritin levels, and NLP-3 inflammasome $[9,11,24,25]$. Multiple other studies also supported decreased oxygen requirements at the end of the treatment period [1,310]. Huet et al showed that oxygen supply decreased to $2 \mathrm{~L} / \mathrm{min}$ from $7 \mathrm{~L} / \mathrm{min}[9]$.

Two studies noticed a significant decrease in temperature reaching normalcy in 2-10 days, improving oxygen requirements and respiratory function $[26,27]$. Two studies noticed a significant improvement in the modified WHO ordinal scale $[10,11]$. Cavalli et al reported improved survival in patients receiving anakinra compared to standard therapy; however, mechanical ventilation-free survival was not significantly improved in the anakinra group than the standard treatment group [10]. Navarro-Millan et al noted that early initiation before requiring supplemental 02 therapy prevented worsening in clinical status (i.e., need for mechanical ventilation). The study also included four mechanically ventilated patients who received anakinra; two patients were discharged, and one patient died [11]. The H-score used to diagnose $\mathrm{HLH}$ was noted to significantly decrease at the end of the treatment period (of anakinra administration to COVID-19 patients) in the studies by Dimopoulos et al and Day et al $[26,27]$. Although both studies reported high sensitivity and specificity for diagnosing $\mathrm{HLH}$, this tool has certain limitations in stratifying between moderate and severe COVID-19 patients, so severity may be underreported [28].

\section{Efficacy Outcome Measurement}

In the studies of Huet et al and Cavalli et al (Figure 3a), the outcome revealed a statistically significant superiority of the anakinra group (13/52) versus the control group with a OR of $0.13(0.05-0.31)$ at $95 \% \mathrm{Cl}$, as well as $(3 / 29)$ versus $(7 / 16)$ with a OR of $0.15(0.03-0.70)$. The pooled results showed an OR of 0.13 $\left(0.06-0.29, p<0.00001, l^{2}=0 \%\right)$.

Subgroup forest plots made for the need for mechanical ventilation in five studies show that Huet et al, 2020 revealed a statistically significant difference favoring anakinra in survival with no need for IMV with OR of $0.13(0.05-0.3, p=0.0005)$. However, no statistically significant difference was noted in Cavalli et al and Narain et al, Dimopoulos et al and Navarro-Millan et al showed the absence of a control group. Pooled results of the studies showed an OR of $0.57(0.11-2.84, p=0.49,12=87 \%)$ (Figure 3b).

Safety outcome for anakinra was measured, indicating 13/81 events (16\%) in anakinra arm in Huet et al with OR of 1.86 (0.58-5.92), and Cavalli et al, 2020 with OR of $0.81(0.12-5.42)$ at $95 \% \mathrm{Cl}$ versus $7 / 60$ events in the control group (11.6\%), with no statistically significant difference between both groups $p=$ 0.46. The pooled outcomes have an OR of 1.48 (0.55-3.99, $p=0.44,12=0 \%)$ (Figure 3c).

Safety outcome measurement as elevated liver transaminases in both arms showed no statistically significant differences between both arms in Huet et al with OR of 1.56 (0.42-5.71), Cavalli et al with OR 
of $0.25(0.05-1.25)$ at $95 \% \mathrm{Cl}, \mathrm{p}=0.08$. Aouba et al and Navarro-Millan et al 2020 showed no control group. The pooled results have an OR of $0.67(0.11-3.93, p=0.66,12=66 \%)$ (Figure 3d).

Anakinra use in COVID-19 patients has shown to have a similar safety profile with the control group without any other significant increase in adverse effects. Cavalli et al, 10 patients in the anakinra group experienced thromboembolic events than 5 in the control group. This was consistent in Huet et al, which showed 3 patients in the anakinra group than 2 in control having thromboembolic events. Cavalli et al, 7 patients in the anakinra group had increased liver transaminase levels than 4 in the control group. This was consistent in Huet et al, which showed 3 patients in the anakinra group than 5 in control. Cavalli et al identified 4 patients with bacteremia in the anakinra group and 2 in the control group. Drug adverse reactions as the clinical outcome were noticed in a retrospective cohort study by Cavalli et al in seven (24\%) patients after a median treatment duration of 9 days (IQR 8-10) with high dose anakinra; four of the patients had bacteremia with the isolation of staphylococcus epidermidis and three had raised serum enzyme levels. This event led to drug discontinuation among patients with a median age of 62 years (IQR 55-71) with at least one comorbidity (arterial hypertension, diabetes, COPD, and tobacco) [10]. No significant adverse events, including bacterial infections, were noted in the study of Huet et al Superimposed infections were most likely seen in mechanically ventilated patients due to respiratory failure. Raise in procalcitonin and transaminase levels were seen in many patients. In another cohort study, raised serum liver aminotransferases were seen [9]. Other complications included injection site reaction and leukopenia in one patient. No drug allergic reactions were seen in any of the patients in the studies. No strong conclusive evidence can be derived from these findings, and more extensive studies with follow-up of patients are required to provide conclusive evidence on the most effective dosing strategies and adverse effects.

\section{Limitation}

The meta-analysis has several limitations. First, none of the included studies were randomized controlled clinical trials. We decided to include a quantitative analysis of the observational studies and qualitative analysis of the case series studies to provide preliminary conclusions for using anakinra in COVID-19 patients. The outcomes in this analysis are derived from a small sample size of patients, resulting in type to error. We could not conduct a meta-analysis on other clinical outcomes due to the incomplete data in the original studies (i.e., mortality in Narain et al). Considering the low certainty of the evidence and varied heterogeneity, it isn't easy to draw a clear conclusion in several outcomes or advantages of anakinra in COVID-19.

\section{Conclusion}

In conclusion, based on the current evidence, anakinra is associated with decreased mortality in COVID19 patients. However, the use of anakinra has not been shown to have a significantly higher safety profile than the control. Many issues, such as patient characteristics, dosage, risk of adverse events, and 
anakinra regimen, remain unclear. Several ongoing clinical trials (NCT04412291; NCT04424056;

NCT04364009; NCT04362943) will help address these issues in the future.

\section{Declarations}

Funding: The authors did not receive any funding for this paper

Conflict of interest: The authors have no conflict of interest to declare

Availability of data and material: The datasets generated during and/or analyzed during the current study are available from the corresponding author on reasonable request.

Authors' contributions: All authors contributed to the design and implementation of the research, including the analysis of results and writing the manuscript.

\section{References}

[1] Vizcarra, P. et al. Description of COVID-19 in HIV-infected individuals: a single-cohort study. Lancet HIV. https://doi.org/10.1016/S2352-3018(20)30164-8 (2020).

[2] CDC. CDC COVID data tracker. CDC. https://covid.cdc.gov/covid-data-tracker (2020).

[3] CDC. Therapeutic options for COVID-19 patients. CDC. https://www.cdc.gov/coronavirus/2019ncov/hcp/therapeutic-options.html (2020).

[4] Lai, M.M.C. \& Holmes, K.V. Coronaviridae: The viruses and their replication. In: Knipe D.M., Howley P.M., editors. "Fields Virology", 4th edn. Lippincott, Williams \& Wilkins; Philadelphia. pp. 1163-1185 (2001).

[5] Esakandari, H. et al. A comprehensive review of COVID-19 characteristics. Biol Proced Online. 22, 19. https://doi.org/10.1186/s12575-020-00128-2 (2020).

[6] Ballet, J.J., Agrapart, M., Durandy, A., Griscelli, C. \& Daguillard, F. Separation of precursor T cells and Igsecreting B cells from the large lymphocytic cells of human tonsil. Cell Immunol. 33, 291-6 (1977).

[7] Mehta, P. et al. COVID-19: consider cytokine storm syndromes and immunosuppression. Lancet. 395, 1033-1034 (2020).

[8] Fardet, L. et al. Development and validation of the HScore, a score for the diagnosis of reactive hemophagocytic syndrome. Arthritis Rheumatol. 66, 2613-2620 (2014).

[9] Huet, T. et al. Anakinra for severe forms of COVID-19: a cohort study. Lancet Rheumatol. 2, e393-e400 (2020). 
[10] Cavalli, G. et al. Interleukin-1 blockade with high-dose anakinra in patients with COVID-19, acute respiratory distress syndrome, and hyper-inflammation: a retrospective cohort study. Lancet Rheumatol. https://doi.org/10.1016/S2665-9913(20)30127-2 (2020).

[11] Navarro-Millán, I. et al. Use of anakinra to prevent mechanical ventilation in severe COVID-19: a case series [published online ahead of print, 2020 Jun 30]. Arthritis Rheumatol. 10.1002/art.41422. https://doi.org/10.1002/art.41422 (2020).

[12] Narain, S. et al. Comparative survival analysis of immunomodulatory therapy for COVID-19 "Cytokine storm": A retrospective observational cohort study. medRxiv, https://doi.org/10.1101/2020.06.16.20126714 (2020)

[13] Zhang, B. et al. Clinical characteristics of 82 cases of death from COVID-19. PLoS One. 15, e0235458 (2020).

[14] Sarfraz, A. et al. Therapeutic plasma exchange and COVID-19: a rapid review. J Clin Immunol Immunother. 6, 041 (2020).

[15] Li, X., Geng, M., Peng, Y., Meng, L. \& Lu, S. Molecular immune pathogenesis and diagnosis of COVID19. J Pharm Anal. 10, 102-108 (2020).

[16] Schulte, W., Bwenhagen, J. \& Bucala, R. Cytokines in sepsis: potent immunoregulators and potential therapeutic targets - an updated view. Mediators of Inflammation. https://doi.org/10.1155/2013/165974 (2013).

[17] Channappanavar, R. \& Perlman, S. Pathogenic human coronavirus infections: causes and consequences of cytokine storm and immunopathology. Semin Immunopathol. 39, 529-539. (2017)

[18] Otsuka, R. \& Seino, K. Macrophage activation syndrome and COVID-19. Inflammation and Regeneration. 40 (2020).

[19] Conti, P. et al. Induction of pro-inflammatory cytokines (IL-1 and IL-6) and lung inflammation by COVID-19: anti-inflammatory strategies. J Biol Regul Homeost Agents. 34(2) pii:1. https://doi.org/10.23812/conti-e (2020).

[20] King, A. et al. Anakinra in COVID-19: important considerations for clinical trials. Lancet Rheumatology. 2, e379-e381 (2020).

[21] Elsevier. Drug Monograph Anakinra. Elsevier. https://covid-19.elsevier.health/en-US/drugmonographs/anakinra (2020).

[22] Medscape. Kineret (Anakinra) dosing, indications, interactions, adverse effects, and more. https://reference.medscape.com/drug/kineret-anakinra-343189\#10 (2020). 
[23] Schett, G., Dayer, J. \& Manger, B. Interleukin-1 function and role in rheumatic disease. Nat Rev Rheumatol. 12, 14-24 (2016).

[24] Pontali, E. et al. Safety and efficacy of early high-dose IV anakinra in severe COVID-19 lung disease. $J$ Allergy Clin Immunol. 146, 213-215 (2020).

[25] Aouba, A. et al. Targeting the inflammatory cascade with anakinra in moderate to severe COVID-19 pneumonia: case series [published online ahead of print, 2020 May 6]. Ann Rheum Dis. 2020;annrheumdis-2020-217706. https://doi.org/10.1136/annrheumdis-2020-217706 (2020).

[26] Dimopoulos, G. et al. Favorable anakinra responses in severe COVID-19 patients with secondary hemophagocytic lymphohistiocytosis. Cell Host Microbe. 28, 117-123 (2020).

[27] Day, J.W. et al. Interleukin-1 blockade with anakinra in acute leukaemia patients with severe COVID19 pneumonia appears safe and may result in clinical improvement. Br J Haematol. 190, e80-e83 (2020).

[28] Leverenz, D.L. \& Tarrant, T.K. Is the HScore useful in COVID-19?. Lancet. 395(10236):e83. https://doi.org/10.1016/S0140-6736(20)31057-6 (2020).

\section{Figures}




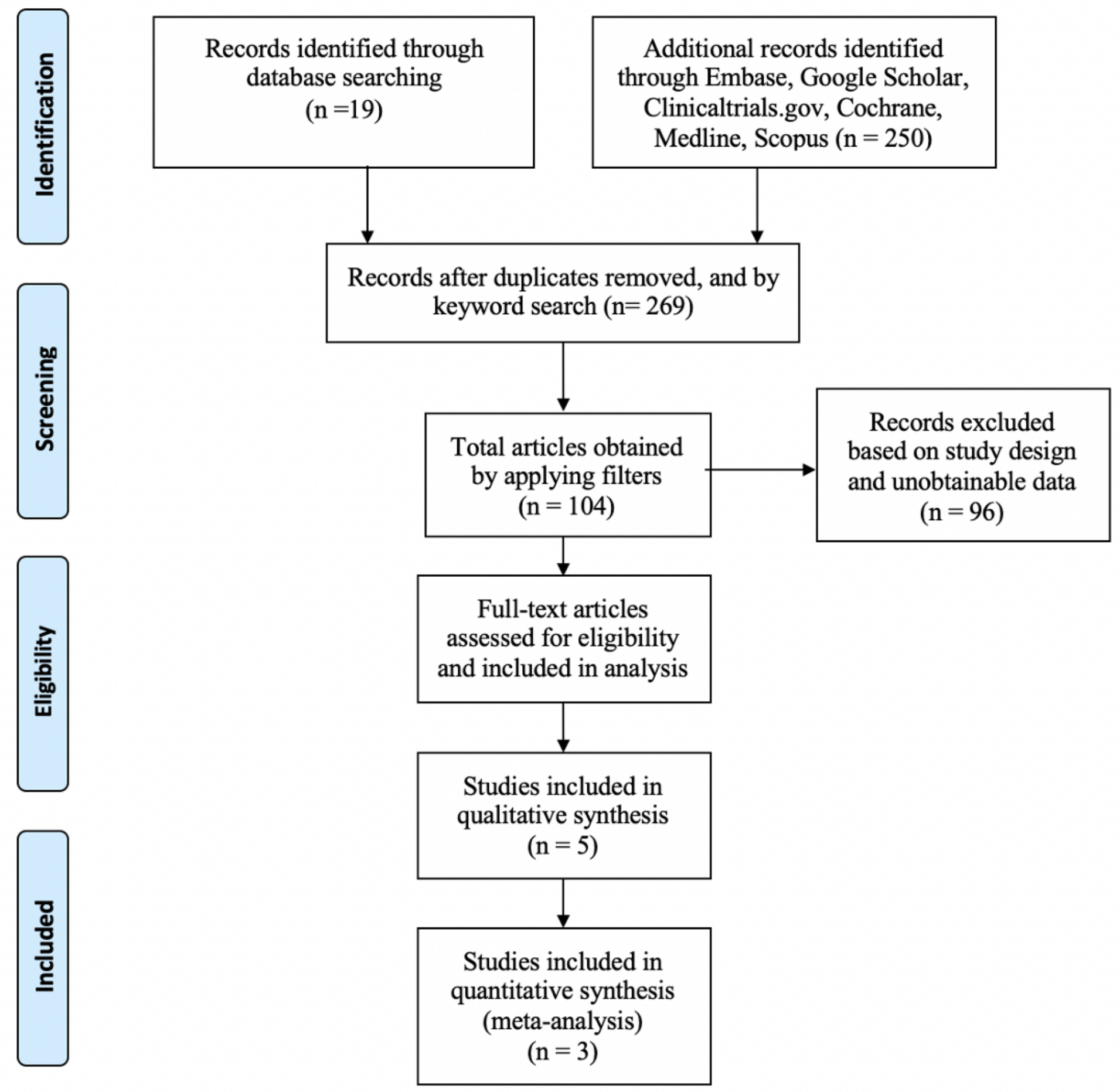

Figure 1

PRISMA flow chart summarizing the literature search. 


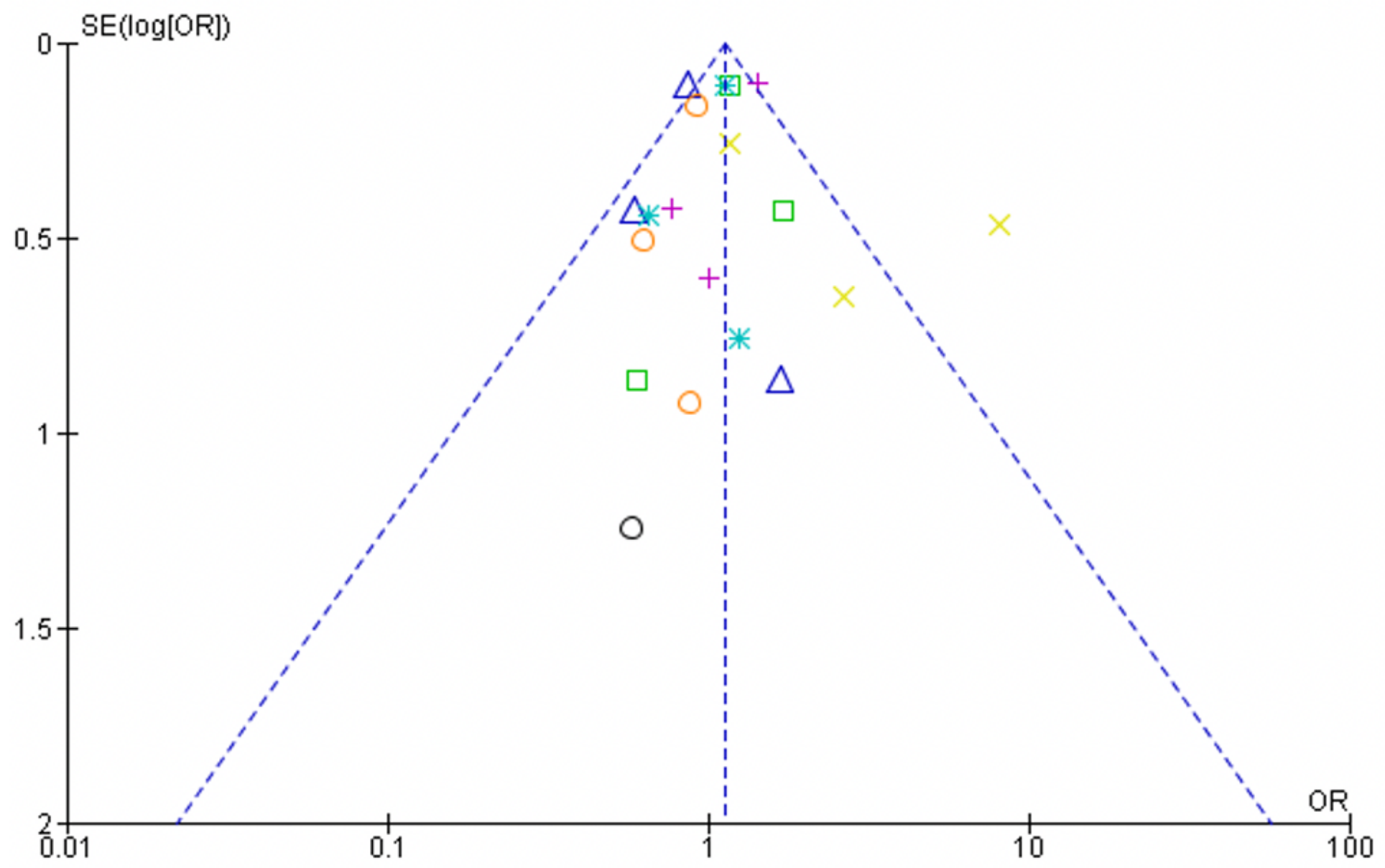

Subgroups

Age $>50$ years old

$\checkmark$ Age $<50$ years old

Male gender

$\triangle$ Female gender

( Survival Outcome (No ICU, Alive, No IMV)

+ Comorbidities Hypertension

* Comorbidities Diabetes

Comorbidities Cardiovascular

\section{Figure 2}

Funnel plot showing no publication bias and high precision among studies. $\mathrm{X}$ axis shows the drug's measured average effect. $Y$ axis shows standard error. 


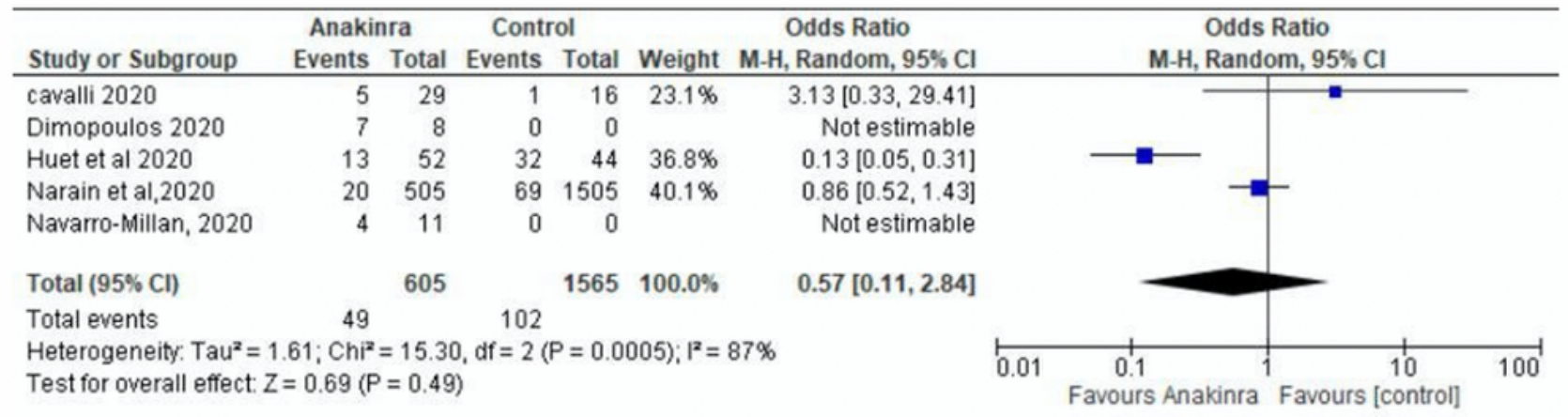

\section{Forestplot A}

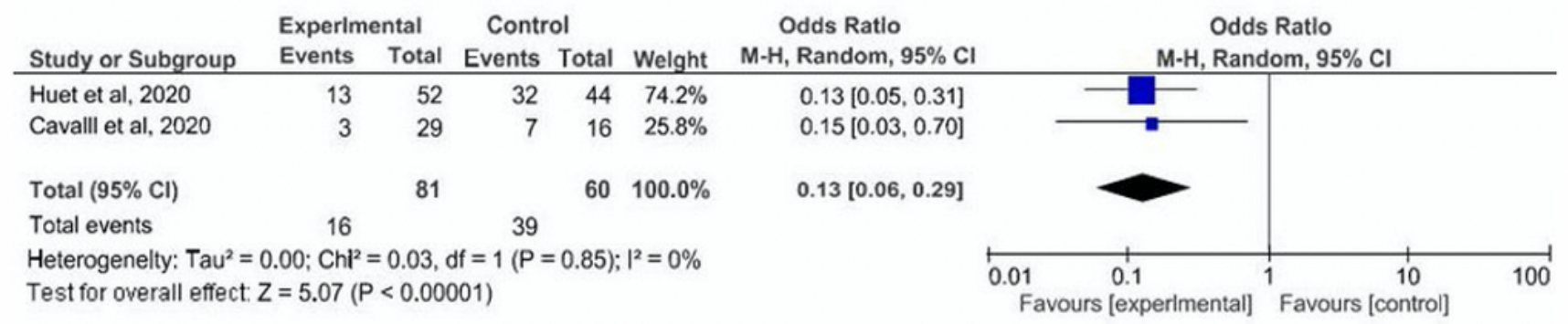

\section{Forestplot B}

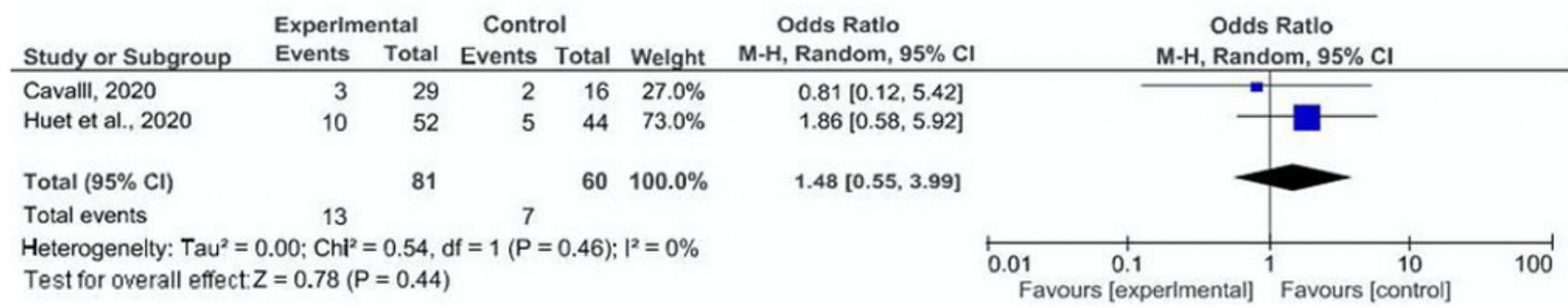

\section{Forestplot C}

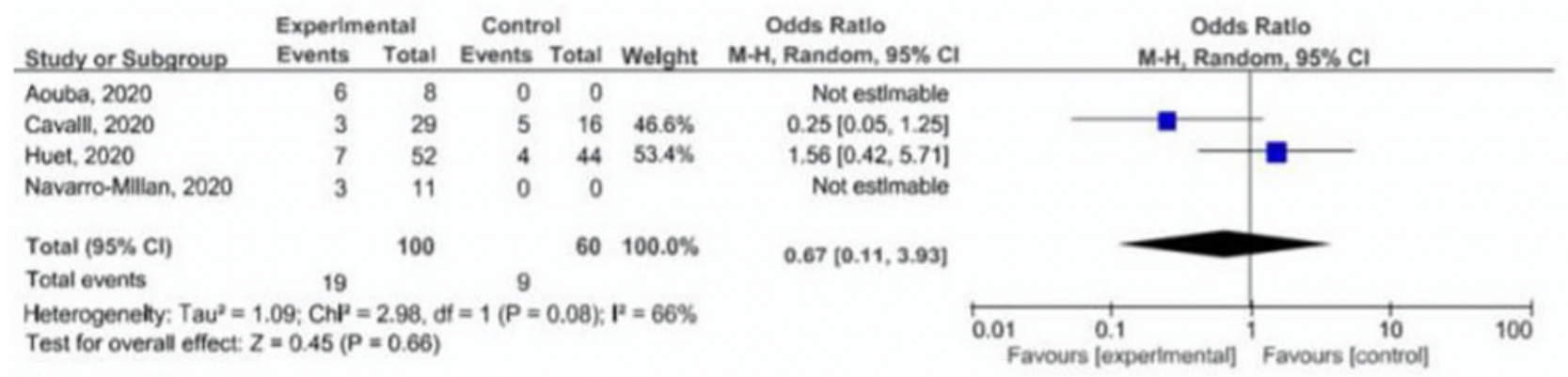

\section{Forestplot D}

\section{Figure 3}

(a) estimates of OR and $95 \% \mathrm{Cl}$ of the primary outcome of mortality rate between Anakinra cohort studies. Summary measure reveals a statistically significant superiority in the experimental group compared to the control. (b) efficacy outcome measured by the need for mechanical ventilation. Estimated odds ratios and confidence intervals of individual and pooled analysis measuring efficacy outcome by the need for mechanical ventilation. Summary measure shows no statistical significance 
between Anakinra and control groups. (c) depicts estimated odds ratio and $95 \%$ confidence intervals of thromboembolic events between experimental and control groups in the 2 included cohort studies.

Summary measure reveals no statistical difference between control and experimental populations. (d) depicts individual and pooled estimates of odds ratios and $95 \%$ confidence intervals of increases in liver transaminases between experimental and control groups. Summary measure shows no statistical difference between experimental and control groups.

\section{Supplementary Files}

This is a list of supplementary files associated with this preprint. Click to download.

- SupplementaryMaterialTable1.docx 